\title{
Computers in psychiatry
}

\section{Linking psychiatric registers to decision support systems}

\author{
JASON TAYLOR, Locum Consultant Psychiatrist, Whittington Hospital, Highgate Hill, \\ London N19
}

This paper discusses the concept of Psychiatric Register Integrated Support Modules (PRISM). It addresses issues around the linkage of longitudinal data held on individual patients in case register systems to expert and other decision support systems. The paper describes two developed systems which link to the SafetyNet case register system, an inexpensive and comprehensive Mental Health Information System which runs on single or networked computers and which was previously described in detail (Taylor \& Bhumgara, 1989). The merits of the two approaches in aiding clinical decision making on anti-psychotic medication are discussed as are the medico-legal implications of using expert systems.

\section{Clinical algorithms in psychiatry}

While there is considerable agreement among psychiatrists regarding the benefits of antipsychotic medication in reducing relapse among patients with schizophrenia, there appear to be no clear guidelines on the rules (clinical algorithms) for altering medication in any given situation. Depot medication review clinics are often conducted by trainees who may have little experience in advising on alterations in depot medication and research has given conflicting advice on optimum dosages (Baldessarini \& Davis, 1980). The problem of advising on an individual case is further hampered by the fact that most trainees who form part of a rotational training scheme will have a limited familiarity with the patients they are seeing in the depot review clinic setting and even when aided by a community psychiatric nurse who has a long-standing knowledge of the patient, the patient's notes may be too bulky or disorganised to develop a clear understanding of treatment and response over the years.

\section{Longitudinal records}

The SafetyNet system builds prospective longitudinal records, creating an active archive, a key feature of case register systems. Assessments within SafetyNet are conducted on reliable, validated scales; the Krawiecka for mental state assessments has been regarded as a sensitive instrument in measuring change in mental state signs in patients with chronic schizophrenia and the Social Behaviour Schedule for rating problems. This allows the recording of changes in mental state and problems for an individual patient to be charted over time. Medication information is also stored with both start and stop dates within an immediately accessible archive within SafetyNet and antipsychotic medication including depot medication information is then converted automatically by the system into chlorpromazine equivalents.

\section{Chloropromazine equivalents}

In a review of research publications exploring the optimum dosages of neuroleptics in schizophrenia, Baldessarini \& Davis (1980) discussed the usage by research workers of estimates of equivalent doses of chlorpromazine. Employing simple formulae, i.e. multiplying a chlorpromazine equivalent ratio by the dosage and frequency of a drug, different anti-psychotic medication can be converted to an equivalent dosage of chlorpromazine per day.

\section{Approaches}

Executive information - time-series graphs

One approach to examining the trends in treatment and response is to plot out selected mental state signs, behavioural problems, interventions, side effects and medication as measured by chlorpromazine equivalents against time. These graphs (e.g. Fig. 1) may be examined for trends exploring the relationships between the variables over many years. The advantage of this technique is that it allows the clinician considerable leeway in decision making but obviates the need for a very time-consuming trudge through case-notes (where information is often disorganised and may be absent).

\section{Linked expert system modules}

APE (anti-psychotic medication expert) is an experimental expert system written under 1st Class 

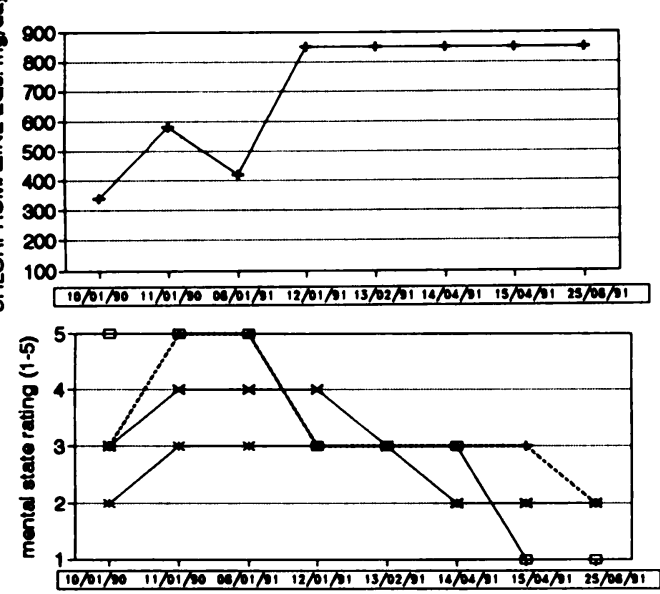

FiG. 1. Plot of selected mental state signs (hallucinations $\nabla$; delusions $\Delta$; anxiety $\Delta$; depression $x$ ) and chlorpromazine equivalents (mg/day) over time.

Fusion, an expert system programming language, that has a link to Dbase IV files. The system aims to offer advice on the use of anti-psychotic medication for an individual patient with a diagnosis of schizophrenia.

\section{What is an expert system?}

Expert systems or 'knowledge-based systems' are computer programs that contain both declarative knowlege (facts about objects, events, and situations) and procedural knowledge (information about courses of action) to emulate the reasoning processes of human experts in a particular domain, or area of expertise.

\section{Fundamental qualities of expert systems}

High level of performance. An expert system must attain the high level of performance that a human expert achieves in some task. It must produce high quality results in a minimum time. However, performance (a difficult dimension to quantify) rather than speed must be regarded as the more important factor.

Efficiency. The quality that all experts possess is the ability to analyse details and arrive at a reasonable hypotheses in a relatively short length of time. This is achieved by the elimination of many possibilities at each inferential stage.

Symbol manipulation. Expert systems employ symbolic reasoning. One factor that distinguishes work on expert systems from simply special-purpose is its relation to Artificial Intelligence in general and to symbolic and representational reasoning.
Heuristic procedures. Experts do not, in general, follow a set of rules. Instead they use heuristics, 'rules of thumb'. They work using available evidence and data, and then ask further questions until they reach a conclusion.

Explanation. All expert systems should be capable of providing clear and concise information about how a conclusion was derived. Explanation in expert systems is usually associated with tracing the rules that are fired during a problem-solving session.

\section{Expert system techniques}

Architecture. One way in which expert sytsems differ from conventional computer programs is that, in general, expert systems separate the declarative knowledge (stored in a knowledge base) from the code that controls the inference and search strategies contained in the system.

Inference and control. The two common control strategies used in rule-based systems are backward and forward chaining. Chaining essentially means to connect knowledge bases together. There are many advantages in using chaining to build expert systems. Firstly, most 'real-world' problems can be naturally broken down into smaller problems. Secondly, modularity is a fundamental concept in the design of any piece of software. It makes development less complicated, and the maintenance, testing, debugging and expansion of the system simpler.

Backward chaining. Backward chaining attempts to find data to prove, or disprove, an hypothesis. The system selects an hypothesis from its knowledge base. It then 'looks' backwards in order to establish which items of evidence it requires in order to resolve that hypothesis.

Forward chaining. Generally, forward chaining involves reasoning from data to hypotheses. This is, therefore, a data-driven strategy. It simply asks a question, or takes what the user gives it, and then makes what inferences it can from that data. It then asks another question, makes inferences and so on until all the hypotheses have been resolved in one way or another.

Anti psychotic medication expert. Some systems may use both forward and backward chaining, or may permit rules that incorporate confidence and probability. APE satisfies the general definition of an expert system in that it was built to perform at the level of an expert to provide advice about the usage medication for individuals suffering from schizophrenia using heuristics defined from clinical 
experience, research findings and psychiatrists' usage of drugs described in medication surveys. APE uses heuristics to derive a conclusion and provides a concise explanation about how this conclusion was reached.

\section{How does APE operate?}

The broad aims of the system are to optimise the level of functioning while minimising medication, simplifying dosage, drug regime and side effects while trying to find medication that is acceptable to the patient.

(a) The clinician selects a patient with a diagnosis of schizophrenia known to SafetyNet.

(b) The clinician selects a set of mental state findings or problems clustered by evidence from recent research examining factor analysis of symptoms.

(c) APE searches for the best ever and current levels (most recent assessment) of functioning on accumulated scores on the selected symptoms for the chosen patient for each assessment. The best ever level of functioning is assessed by the system looking for lowest cumulated scores from ratings which have remained stable over a defined period of time or number of sequential assessments when the medication has been lowest (as measured by chlorpromazine equivalents). APE then provides feedback on screen of the current level of functioning as a percentage of best ever level of functioning.

(d) APE calculates the chlorpromazine equivalents at those two times (best and current assessment dates).

(e) The system then offers advice on changes in dosage as measured by chlorpromazine equivalents based on level of functioning and on a series of defined in-built rules relating to the current symptoms and the recent trends in symptomatology (i.e. patient improving, stable or deteriorating), current side effects, number of admissions, date of last admission, compliance, patient's attitude to change in medication etc.

(f) APE finally offers advice on specific medication based on inbuilt rules relating to current treatment, patient satisfaction, simplification of drug regime etc.

(g) Throughout, the system aims to feedback the information gleaned from SafetyNet and the path that APE took through the rules in order to achieve its advice; thus at each stage in the decision making process giving the user feedback on how it reached its conclusions.

\section{Law, liability and expert systems}

Expert systems - a legal definition. A discussion on liability and responsibility for expert systems should be based upon a clear definition of the nature of an expert system. There is much discussion and disagreement as to the formal definition of an expert system. Susskind (1986) advocated that "only computers capable of performing at the level of experts in a given domain are worthy of the appellature expert systems."

It may be argued that the legal understanding of what an expert system is, should be based upon what society considers an 'expert' to be.

System access. In order to reduce the issue of legal liability and responsibility for the consequences of the use of expert systems it must be clarified who will actually have access to the system. Totally unrestricted access to the system would considerably broaden the area of liability. It is suggested that a distinction should be made between the use of expert systems which are restricted to 'professionals' and the situation where there is essentially unrestricted use. This has particular implications in tort liability and two relevant off-shoots, strict liability (i.e. liability without fault) and professional malpractice. It is possible that a situation may arise where an expert system is marketed as 'off the shelf and as such the program would constitute a product, and, therefore, fall under the category of strict liability rather than that of professional malpractice.

Liability. Phillip Leith reports that 'With large amounts of litigation surrounding medical malpractice in the USA, problems about legal responsibility for bad advice have not been cleared up; are the inventors of the system legally liable for damages arising from malpractice?' (Card \& James, 1990).

Entrepreneurs are in general unwilling to market products that may perhaps result in a wave of liability suits rather than a profitable return on outlay. It is believed that this, and the fact that the issue of liability for expert systems is an unclear area of law, may impede the actual development of such systems.

There are some important implications of liability.

(a) To what extent is the system-user liable for reliance on the system?

(b) To what extent is the system-user liable for failure to consult the system?

(c) To what extent is the designer of the system liable to faults in the system design?

(d) To what extent are the experts whose knowledge is embodied in the system liable for any mistakes in the knowledge supplied?

Actions for liability in the law are based upon society's understanding of a 'standard of care'. The general principle is that a person is normally held liable only if it can be proved that his conduct fell short of this general objective standard. The professional is expected to make such enquiries as are 
necessary to reach a professional judgement that is not a considered conclusion.

It is accepted that when a professional is faced with a problem outside his field of expertise he is expected to consult an expert. This expert may be an expert system. A consequence of consulting the expert, but failing to conform with the advice given by the expert (in this case an expert system), may lead to a situation whereby plaintiffs base claims on the fact that the expertise of available expert systems is greater than that of the professional. The Law Society confirms that there are no, or at least few, specific laws governing the development of expert systems. There have to their knowledge been no cases involving liability with regard to expert systems in this country. They suggested that either the programmer or the doctor could be liable 'depending upon the specific circumstances.'

\section{Problems with PRISM}

There are many problems associated with linking to data held in psychiatric case registers. Information is time-consuming to collect, may not get input regularly or accurately and data may also show poor inter-rater reliability. These criticisms can, however, also be justifiably applied to clinical notes. The more difficult issues are those regarding the validity of the clinical algorithms which because of a limited understanding of many of the issues around the use of anti-psychotic medication and chlorpromazine equivalents ensure that the APE system is a highly experimental prototype. Medico-legal issues and clinicians' attitudes may also limit the use of expert systems in clinical care.

\section{Contrasting approaches}

The two approaches detailed are different in the degree of control they offer the clinician in the decision making process. The time series graphs allow considerable control in decision making but do not help the clinician to clarify the rules by which (s)he has reached a decision. The advantage of an expert system approach is that the decision making process can be described at each stage and this challenges the clinician to accept or dispute the advice (based on the data within the system) and may help the clinician to evaluate his/her own decision-making process, to make his/her "clinical algorithms" explicit to justify his/her reasoning. Overall, the main value of successful executive information systems and decision support systems may be their usefulness in providing motivation for clinicians "rewarding" them for the efforts of data entry.

\section{Evaluating systems}

The two approaches described will need to be evaluated and the expert system in particular will need a staged evaluation. Initially, one would need to explore the safety of the advice (although the system warns on dosages above the BNF recommendations) and before use in a clinical setting evaluate the system through the use of blind comparisons by independent psychiatrists contrasting aided with unaided clinicians' medication choices. It remains to be seen whether such systems can have any effect on the longer term outcome or on how psychiatrists think about the process of clinical decision-making.

\section{Acknowledgements}

I wish to express thanks to Miss Sharon Berry, Computer Scientist, for programming work contributing towards the development of the APE system. I also wish to thank sponsors of the SafetyNet Project, in particular Marks \& Spencer and Friern Trustees.

\section{References}

Baldessarini, R. J. \& Davis, J. M. (1980) What is the best dose of neuroleptics in schizophrenia? Psychiatry Research, 3, 115-122.

CARD, R. \& JAMES, J. (1990) Law for Accountancy Students. London: Butterworths.

SUSSKIND, R. E. (1986) Expert systems in law: a jurisprudential approach to artificial intelligence and legal reasoning. Modern Law Review, 49, 178.

TAYLOR, J. \& BhumgarA, K. (1989) The SafetyNet Project. Psychiatric Bulletin, 13, 677-679.

A full list of references is available on request to the author. 